\title{
Violation of the London law and Onsager-Feynman quantization in multicomponent superconductors
}

\author{
EGOR BABAEV ${ }^{1,2 *}$ AND N. W. ASHCROFT² \\ ${ }^{1}$ Department of Theoretical Physics, The Royal Institute of Technology, 10691 Stockholm, Sweden \\ ${ }^{2}$ Laboratory of Atomic and Solid State Physics, Cornell University, Ithaca, New York 14853, USA \\ *e-mail: babaev@kth.se
}

Non-classical response to rotation is a hallmark of quantum ordered states such as superconductors and superfluids. The rotational responses of all currently known single-component 'super' states of matter (superconductors, superfluids and supersolids) are largely described by two fundamental principles and fall into two categories according to whether the systems are composed of charged or neutral particles: the London law $^{1}$ relating the angular velocity to a subsequently established magnetic field and the Onsager-Feynman quantization of superfluid velocity ${ }^{2,3}$. These laws are theoretically shown to be violated in a two-component superconductor such as the projected liquid metallic states of hydrogen and deuterium at high pressures. The rotational responses of liquid metallic hydrogen or deuterium identify them as a new class of dissipationless states; they also directly point to a particular experimental route for verification of their existence.

For superfluid systems composed of electrically neutral particles (liquids, vapours or even solids ${ }^{4}$ ) and for slow rotations, a fraction of the system, the superfluid fraction, remains irrotational. However, in response to rotation above a certain critical rotation frequency, the superfluid fraction comes into rotation by means of vortex formation. Onsager ${ }^{2}$ and Feynman ${ }^{3}$ pointed out that the superfluid velocity $(\mathbf{v})$ in these vortices in single-component systems is quantized, and the circulation quantum $K$ depends only on the particle's mass $m$ and Planck's constant $\hbar: K=(1 / 2 \pi) \oint \mathbf{v} \cdot \mathrm{d} \mathbf{l}=\hbar / m$. For superfluids with, for example, $p$-wave symmetry of the order parameter, which are also invariant under simultaneous phase and spin transformations, this quantization is modified ${ }^{5}$. A special situation also occurs in a mixture of superfluids, where a superfluid velocity of one condensate can drag the superfluid density of another ${ }^{6,7}$.

For systems composed of charged particles that are also superconducting, vortices are not induced by rotation. London showed that a uniformly rotating single-component superconductor generates a persistent current in a thin layer near its surface, and this in turn produces a detectable magnetic field, the London field ${ }^{1}$. London related this field to the rotation frequency, $\boldsymbol{\Omega}$, according to $\mathbf{B}=-(2 m c / e) \boldsymbol{\Omega}$, where $m$ is the electron's mass, $e$ denotes its electric charge and $c$ is the speed of light. This law is experimentally confirmed (see for example ref. 8). Of crucial significance is the fact that the experimentally observed London law involves only fundamental constants, and not materials properties specific to the superconductor (such as an effective mass for electrons). This law also holds with no modifications for electronic superconductors with $d$ - and $p$-wave pairing symmetry.

We consider the responses to rotation of the projected novel quantum states with two electrically charged components showing off-diagonal long-range order and that are now the subjects of renewed experimental pursuit. Liquid metallic states of hydrogen were predicted earlier to show Cooper pairing in both protonic and electronic channels ${ }^{9}$; however, it should be noted that an even simpler situation may occur in liquid metallic deuterium because deuterons are bosons and can undergo condensation without the need for a pairing instability. Also in a hydrogen-rich alloy under extreme but experimentally accessible pressures both electrons and protons may be mobile in a crystalline lattice ${ }^{10}$. Finally, a rotational response similar to that discussed below would be present in solid metallic hydrogen and deuterium if they possessed a metallic equivalent of supersolidity. For brevity below we shall always refer to 'liquid metallic hydrogen (LMH)', but it is important to keep in mind that the range of potential applications is much wider, including recent discussions of possible presence of several charged baryonic condensates in neutron stars ${ }^{11}$. Previous studies of these states have, however, mostly focused on the reaction of the system to an applied magnetic field ${ }^{12,14,15}$; here, our intention is to study the reaction of the system to rotation.

In the hydrodynamic approach a two-component superconductor is described by the following free energy:

$$
F=\sum_{\alpha=\mathrm{e}, \mathrm{p}} \frac{1}{2 m_{\alpha}}\left|\Psi_{\alpha}\right|^{2}\left(\nabla \theta_{\alpha} \pm e \mathbf{A}\right)^{2}+\frac{(\nabla \times \mathbf{A})^{2}}{2} .
$$

Here, $\Psi_{\alpha}=\left|\Psi_{\alpha}\right| \mathrm{e}^{i \theta_{\alpha}}$ and $m_{\alpha}(\alpha=\mathrm{e}, \mathrm{p})$ denote electronic and protonic condensate wavefunctions and corresponding masses and A is the vector potential. In this work we focus on the effects caused by the coupling to the gauge field and thus we do not consider possible drag effects ${ }^{6}$, nor do we consider different pairing symmetries. In what follows we set $\hbar=1, c=1$. This model can be rewritten as

$$
\begin{aligned}
F= & \frac{1}{2} \frac{\frac{\left|\Psi_{\mathrm{e}}\right|^{2}}{m_{\mathrm{e}}} \frac{\left|\Psi_{\mathrm{p}}\right|^{2}}{m_{\mathrm{p}}}}{\frac{\left|\Psi_{\mathrm{e}}\right|^{2}}{m_{\mathrm{e}}}+\frac{\left|\Psi_{\mathrm{p}}\right|^{2}}{m_{\mathrm{p}}}}\left(\nabla\left(\theta_{\mathrm{e}}+\theta_{\mathrm{p}}\right)\right)^{2}+\frac{1}{2} \frac{1}{\frac{\left|\Psi_{\mathrm{e}}\right|^{2}}{m_{\mathrm{e}}}+\frac{\left|\Psi_{\mathrm{p}}\right|^{2}}{m_{\mathrm{p}}}} \\
& \times\left(\frac{\left|\Psi_{\mathrm{e}}\right|^{2}}{m_{\mathrm{e}}} \nabla \theta_{\mathrm{e}}-\frac{\left|\Psi_{\mathrm{p}}\right|^{2}}{m_{\mathrm{p}}} \nabla \theta_{\mathrm{p}}-e \mathbf{A}\left[\frac{\left|\Psi_{\mathrm{e}}\right|^{2}}{m_{\mathrm{e}}}+\frac{\left|\Psi_{\mathrm{p}}\right|^{2}}{m_{\mathrm{p}}}\right]\right)^{2}+\frac{\mathbf{B}^{2}}{2} .
\end{aligned}
$$


The first term here shows no coupling to the gauge field and therefore represents a neutral (or superfluid) mode, which is associated with codirected flows of electronic and protonic Cooper pairs (with no net charge transfer) ${ }^{12}$. The second term accounts for the superconducting (or charged) sector of the model describing electrical currents. In what follows, we denote a vortex with phase windings $\left(\Delta \theta_{\mathrm{e}}=2 \pi n_{\mathrm{e}}, \Delta \theta_{\mathrm{p}}=2 \pi n_{\mathrm{p}}\right)$ by a pair of integers $\left(n_{\mathrm{e}}, n_{\mathrm{p}}\right)$.

We begin with inspection of the composite neutral mode's response to rotation. The simplest topological excitation in the superfluid sector of the model (that is, a simplest vortex that has a non-trivial winding in the phase sum $\left.\left(\theta_{\mathrm{e}}+\theta_{\mathrm{p}}\right)\right)$ is a vortex with the windings of only one of the phases: $( \pm 1,0)$ or $(0, \pm 1)$. We note that because the first term in (1) is symmetric with respect to electronic and protonic condensates both the $(1,0)$ vortex and the $(0,1)$ vortex have identical configurations of the neutral composite (that is, consisting of both electrons and protons) superflow. The difference between these two vortices lies only in the contribution to the second term in (1), representing the charged (superconducting) sector of the model.

We first focus on a $(0,1)$ vortex. For this case, the solution for vector potential $\mathbf{A}$ at distances from the core much larger than penetration length is given by ${ }^{12}$ $|\mathbf{A}|=(1 /|e| r)\left(\left|\Psi_{\mathrm{p}}\right|^{2} / m_{\mathrm{p}}\right)\left[\left(\left|\Psi_{\mathrm{p}}\right|^{2} / m_{\mathrm{p}}\right)+\left(\left|\Psi_{\mathrm{e}}\right|^{2} / m_{\mathrm{e}}\right)\right]^{-1}$, where $r$ is the distance from the core centre. The superfluid velocities of electrons and protons in such a vortex at a large distance from the core are $\mathbf{v}_{\mathrm{p}}=\left(\nabla \theta_{\mathrm{p}}+e \mathbf{A}\right) / m_{\mathrm{p}}$ and $\mathbf{v}_{\mathrm{e}}=-e \mathbf{A} / m_{\mathrm{e}}$. An equilibrium of a rotating system is achieved when the quantity $E_{r}=E-\mathbf{M} \cdot \boldsymbol{\Omega}$ is minimal ( $\boldsymbol{\Omega}$ is the rotation frequency and $\mathbf{M}$ and $E$ are the angular momentum and energy). Observe that if a system nucleates a $(1,0)$ vortex then not only protons but also electrons contribute to the angular momentum, whose magnitude is given by $|\mathbf{M}|=\left|\mathbf{M}_{\mathrm{p}}+\mathbf{M}_{\mathrm{e}}\right|=\int\left(m_{\mathrm{p}}\left|\Psi_{\mathrm{p}}\right|^{2} v_{\mathrm{p}}+m_{\mathrm{e}}\left|\Psi_{\mathrm{e}}\right|^{2} v_{\mathrm{e}}\right) r \mathrm{~d} V$.

The superfluid velocity circulations for protons and electrons in a $(0,1)$ vortex are given by $\oint \mathbf{v}_{(\mathrm{e}, \mathrm{p})} \cdot \mathrm{d} \mathbf{l}=2 \pi K_{(\mathrm{e}, \mathrm{p})}=$ $2 \pi\left(\left|\Psi_{(p, e)}\right|^{2} / m_{(p, e)}\right)\left[\left(\left|\Psi_{\mathrm{p}}\right|^{2} / m_{\mathrm{p}}\right)+\left(\left|\Psi_{\mathrm{e}}\right|^{2} / m_{\mathrm{e}}\right)\right]^{-1}\left(1 / m_{(\mathrm{e}, \mathrm{p})}\right)$. From this we observe that in the two-component superconductor the Onsager-Feynman quantization rule is violated: the superfluid velocity quantization is fractional and the electronic and protonic circulation quanta $K_{\mathrm{e}, \mathrm{p}}$ depend not only on mass but also on densities according to

$$
K_{\mathrm{e}}=\frac{\frac{\left|\Psi_{\mathrm{p}}\right|^{2}}{m_{\mathrm{p}}}}{\frac{\left|\Psi_{\mathrm{p}}\right|^{2}}{m_{\mathrm{p}}}+\frac{\left|\Psi_{\mathrm{e}}\right|^{2}}{m_{\mathrm{e}}}} \frac{1}{m_{\mathrm{e}}} ; \quad K_{\mathrm{p}}=\frac{\frac{\left|\Psi_{\mathrm{e}}\right|^{2}}{m_{\mathrm{e}}}}{\frac{\left|\Psi_{\mathrm{p}}\right|^{2}}{m_{\mathrm{p}}}+\frac{\left|\Psi_{\mathrm{p}}\right|^{2}}{m_{\mathrm{e}}}} \frac{1}{m_{\mathrm{p}}} .
$$

The quantization conditions (2) also hold for the vortex $(1,0)$. It has been argued previously that quantization of magnetic flux in $\mathrm{LMH}$ is also fractional ${ }^{12}$. The fractionalization of superfluid velocity quantization that we find here has, however, a different pattern. To compare the fractionalization of the magnetic flux quantum $\Phi_{0}=2 \pi / e$ and the fractionalization of superflow quantization we introduce an angle $\beta$ as a measure of the ratio of the average condensate densities as follows: $\sin ^{2}(\beta / 2)=\left(\left|\Psi_{\mathrm{e}}\right|^{2} / m_{\mathrm{e}}\right)\left[\left(\left|\Psi_{\mathrm{p}}\right|^{2} / m_{\mathrm{p}}\right)+\left(\left|\Psi_{\mathrm{e}}\right|^{2} / m_{\mathrm{e}}\right)\right]^{-1}$; $\cos ^{2}(\beta / 2)=\left(\left|\Psi_{\mathrm{p}}\right|^{2} / m_{\mathrm{p}}\right)\left[\left(\left|\Psi_{\mathrm{p}}\right|^{2} / m_{\mathrm{p}}\right)+\left(\left|\Psi_{\mathrm{e}}\right|^{2} / m_{\mathrm{e}}\right)\right]^{-1}$. Let $K_{(\mathrm{e}, \mathrm{p})}^{0}=$ $1 / m_{(\mathrm{e}, \mathrm{p})}$ be the standard superflow circulation quantum in a one-component neutral superfluid composed of particles with the masses of electronic and protonic Cooper pairs correspondingly. The quantization fractionalization pattern in this notation is then summarized in the Table 1.

The energy per unit length $E_{0}$ of vortices $(1,0)$ and $(0,1)$ contains a logarithmically divergent part arising from the first term in (1) (refs 12,13):

$$
E_{0} \approx \pi\left[\sin ^{4}\left(\frac{\beta}{2}\right) \frac{\left|\Psi_{\mathrm{p}}\right|^{2}}{m_{\mathrm{p}}}+\cos ^{4}\left(\frac{\beta}{2}\right) \frac{\left|\Psi_{\mathrm{e}}\right|^{2}}{m_{\mathrm{e}}}\right] \log \frac{R}{a},
$$

Table 1 Fractionalization of superflow circulation and magnetic flux quanta.

\begin{tabular}{llr}
\hline Vortex & $(1,0)$ & $(0,1)$ \\
\hline Magnetic flux & $\sin ^{2}(\beta / 2) \Phi_{0}$ & $-\cos ^{2}(\beta / 2) \Phi_{0}$ \\
Electronic superflow circulation & $\cos ^{2}(\beta / 2) K_{\mathrm{e}}^{0}$ & $\cos ^{2}(\beta / 2) K_{\mathrm{e}}^{0}$ \\
Protonic superflow circulation & $\sin ^{2}(\beta / 2) K_{\mathrm{p}}^{0}$ & $\sin ^{2}(\beta / 2) K_{\mathrm{p}}^{0}$ \\
\hline
\end{tabular}

where $a$ is a cut-off length that depends on the core structure and $R$ is the distance from the vortex centre to the system boundary. The formation of vortices in response to rotation is controlled by the neutral mode (that is, by the first term in (1)). As discussed above, the vortices $(1,0)$ and $(0,1)$ have the same neutral superflow but different contributions to the second term in (1). The energetically preferred excitations forming in response to rotation are therefore the $(0,1)$ vortices, which carry a smaller fraction of $\Phi_{0}$.

A vortex $(0,1)$ placed into a cylindrical system with radius $R$ and unit height has an angular momentum $|\mathbf{M}|=\pi R^{2}\left(\left|\Psi_{\mathrm{e}}\right|^{2} / m_{\mathrm{e}}\right)\left(\left|\Psi_{\mathrm{p}}\right|^{2} / m_{\mathrm{p}}\right)\left[\left(\left|\Psi_{\mathrm{p}}\right|^{2} / m_{\mathrm{p}}\right)+\left(\left|\Psi_{\mathrm{e}}\right|^{2} / m_{\mathrm{e}}\right)\right]^{-1}$ $\left(m_{\mathrm{e}}+m_{\mathrm{p}}\right)$. Vortices form when $E_{r}=E_{0}-\mathbf{M} \cdot \boldsymbol{\Omega}<0$. This determines the critical rotation frequency as

$$
\Omega_{\mathrm{c}} \approx \frac{1}{R^{2}\left(m_{\mathrm{e}}+m_{\mathrm{p}}\right)} \log \frac{R}{a} .
$$

We can make a rough estimate of critical frequency: $\Omega_{\mathrm{c}} \approx\left(m_{\mathrm{e}} / m_{\mathrm{p}}\right)\left(e^{2} / a_{0}\right)\left(a_{0} / R\right)^{2} \log (R / a)$, where $a_{0}$ is the Bohr radius, which for a $100 \mu \mathrm{m}$ sample is of the order of $10 \mathrm{~Hz}$. Note that from the above considerations it follows that in a similar system but composed of two types of particle with equal masses and charges (such as electronic superconductors where multicomponent order parameters arise from non-s-wave pairing symmetry) no vortices can be induced by rotation.

A quite deep difference in the rotational physics in twocomponent charged systems is manifested especially in the novel 'aggregate states' of vortex matter they should allow. As discussed above, in the simplest case the rotating system forms a lattice of $(0,1)$ vortices (see Fig. 1a). The most interesting situation arises when a rotating system is also subjected to a magnetic field. In this case the possible states of vortex matter are numerous, and we will consider here some particularly interesting possibilities of novel states of 'vortex matter'.

If a weak magnetic field is applied in a direction opposite to the field of rotation-induced vortices, the superconducting sector of (1) will try to minimize its energy by introducing $(1,-1)$ vortices. These vortices have no neutral superflow (electronic and protonic currents are counter-directed) but carry one magnetic flux quantum $^{12}$. However, a vortex $(1,-1)$ is not stable in a lattice of $(0,1)$ vortices because it experiences an attraction to such vortices within the range of the penetration length scale ${ }^{13}$. A $(1,-1)$ vortex therefore should annihilate with a $(0,1)$ vortex, resulting in a $(1,0)$ vortex state. At length scales larger than the penetration length a $(1,0)$ vortex has a Coulomb repulsive interaction with a $(0,1)$ vortex similar to interaction between two $(0,1)$ vortices $^{13}$, and therefore under normal conditions will occupy a space in a rotation-induced lattice of $(0,1)$ vortices; it can therefore be viewed as a ground-state 'electronic vortex impurity' in a 'protonic vortex lattice' (see Fig. 1b). The concentration of these 'vortex impurities' depends on the applied magnetic field and there are indeed many interesting possibilities for their orderings and phase transitions.

Consider next a situation with a stronger magnetic field and with a rotation frequency just above $\Omega_{\mathrm{c}}$. Then the dominant structure is a field-induced lattice of composite $(1,-1)$ vortices (as in the case of no rotation ${ }^{12,14,15}$ ). Here the energetically most 

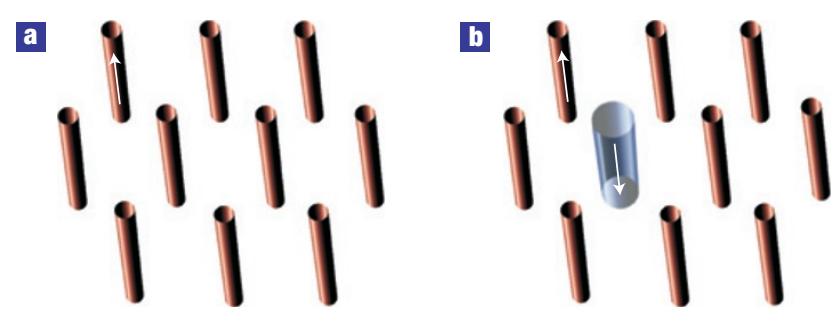

G

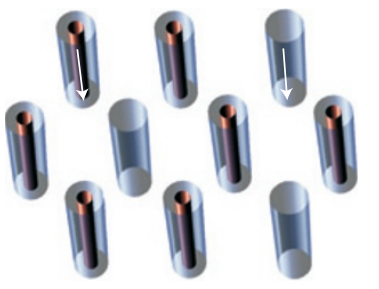

d

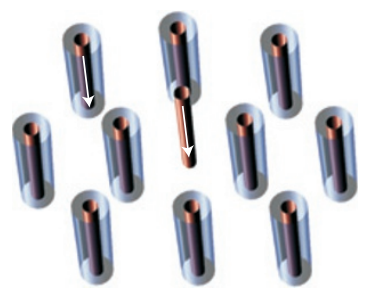

Figure 1 Vortices in a two-component superconductor. a, A rotating two-component superconductor forms a lattice of 'protonic' $(0,1)$ vortices (small red tubes). The arrow denotes the direction of the self-induced magnetic field. $\mathbf{b}$, When an external field is applied in the direction opposite to the field carried by rotation-induced vortices, the ground state possesses $(1,0)$ 'electronic vortices' (blue tubes) placed as 'impurities' in the protonic vortex lattice. c,d, In a strong magnetic field, and for rotations just above the critical frequency, the ground state features either 'electronic vortex impurities' in the lattice of composite vortices or 'interstitial' protonic vortices, depending on the applied field direction.

favourable way to introduce a superfluid momentum-carrying vortex is the substitution of one of the $(1,-1)$ vortices by a $(1,0)$ vortex (see Fig. 1c). This vortex interacts repulsively with its neighbours and carries almost one magnetic flux quantum, but also possesses angular momentum in the superfluid sector. It is therefore an 'elementary vortex impurity' in a lattice of composite vortices. Such a system should show a number of novel phase transitions and vortex-matter states. One such transition will occur because there is a finite potential barrier for a 'vortex impurity' to jump from one lattice site to another. At certain temperatures the vortex impurities should be able to move from one site to another freely. There is an analogy between 'light' vortices, which in the ground state are concentred with 'heavy' vortices, and a system of Bose particles living on sites of a triangular lattice, which is known to possess a supersolid state (see for example ref. 16). In this analogy the $z$ direction in the vortex lattices plays the role of 'time' in the Bose system and the 'heavy' vortices play the role of a robust 'lattice potential'. There arises therefore the possibility of an interesting 'aggregate state of vortex matter', analogous to the supersolid state of Bose particles, which in our case may be called a 'vortex supersolid'. This state should feature coexistence of crystalline order of vortices and 'fluidity' of 'vortex impurities'. Because of the fluid state of light vortices the phase of the corresponding condensate will be disordered in the $z$ direction, which in turn should destroy the order in gaugeinvariant phase sum. For this reason the transition into this 'vortex supersolid' phase has an important physical consequence, namely, the disappearance of superfluidity of particles along the rotation axis, which constitutes the phase transition from superconducting superfluidity to superconductivity selectively along this direction.

In the case where the rotation direction is inverted, while the magnetic field is unchanged, the topological defects in the superfluid sector that minimize energy in a rotating frame are $(-1,0)$ and $(0,-1)$. The former vortex subtracts almost one

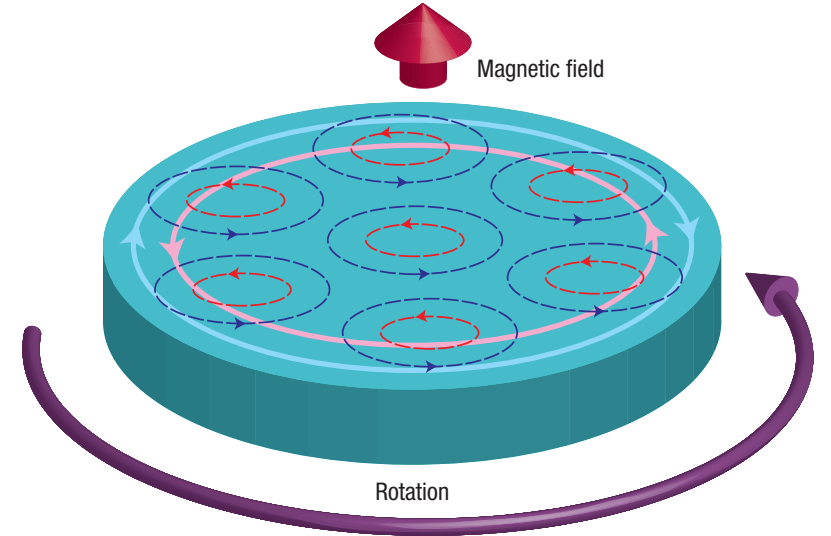

Figure 2 Rotating two-component superconductor. If a two-component superconductor is brought into rotation two types of particle flow are generated: for slow rotation the two components (here electrons and protons) start circulating in opposite directions (schematically denoted by thick pink and light-blue arrows), inducing a magnetic field $\mathbf{B}$ along the rotation axis. At a faster rotation a second type of particle flow also appears in the form of quantum vortices. Here electrons and protons flow in the same direction (thin dashed red and blue arrows). In this picture the basic laws governing rotational response of one-component quantum fluids, the Onsager-Feynman quantization of superflow circulation and the London law, are violated.

magnetic flux quantum and should be compensated by two $(1,-1)$ vortices. In this scenario a lattice should become more dense (with a certain energy penalty). On the other hand there is a second possibility to acquire angular momentum: the introduction of a $(0,-1)$ vortex. This results in a different type of energy penalty: a vortex $(0,-1)$ interacts via a screened potential with a composite vortex $(1,-1)$ but the interaction strength is weaker than between two composite vortices ${ }^{13}$. Therefore, such a vortex can be introduced as an 'interstitial vortex defect' (see Fig. 1d), which, for a range of parameters, should be a more energetically favourable way to acquire angular momentum than the first possibility. The 'light' vortices may form an 'interstitial vortex liquid' state, whereas the concentricity of light vortices with the lattice of heavy vortices is controlled by a different energy scale. This is again a state with coexistent vortex crystalline order and vortex defect fluidity and yet another example of a 'vortex supersolid', which resembles the supersolid state of interstitial particles in crystals discussed in ref. 4.

Finally, let us discuss the reaction of the superconducting sector of the system to rotation. It is important to note that electronic and protonic Cooper pair momenta depend on the same vector potential, $\mathbf{P}_{\alpha} \equiv \nabla \theta_{\alpha}=m_{\alpha} \mathbf{v}_{\alpha}+e_{\alpha} \mathbf{A}$ and hence $\mathbf{A}=\left(\mathbf{P}_{\alpha} / e_{\alpha}\right)-$ $\left(m_{\alpha} / e_{\alpha}\right) \mathbf{v}_{\alpha}$ (where $\left.e_{(\mathrm{e}, \mathrm{p})}= \pm e\right)$. Consider now the situation without an applied external field and low rotation frequencies, so that there are no vortices (that is, $\Omega<\Omega_{\mathrm{c}}$ ). Then, taking the curl of the previous expression, we arrive at the constraint dictated by gauge invariance:

$$
\frac{m_{\mathrm{p}}}{e_{\mathrm{p}}} \nabla \times \mathbf{v}_{\mathrm{p}}=\frac{m_{\mathrm{e}}}{e_{\mathrm{e}}} \nabla \times \mathbf{v}_{\mathrm{e}} .
$$

Turn first to the zero-temperature $(T=0)$ case when there is no normal component. If the condensate charges entering the problem are opposite (as is indeed the case for $\mathrm{LMH}$ ) this equation has a trivial solution: $\mathbf{v}_{\mathrm{p}}=\mathbf{v}_{\mathrm{e}}=0$; that is, at $T=0$ for $\Omega<\Omega_{\mathrm{c}}$ the condensates remain irrotational. However, in the presence of a 
normal component with a net electric charge its rotation produces an electric current, so $\mathbf{v}_{\mathrm{p}}=\mathbf{v}_{\mathrm{e}}=0$ can no longer be a stationary solution. From (3) it also follows that, in contrast to London's picture for ordinary superconductors ${ }^{1}$, superconducting electrons and protons will not follow the rotation of the normal component because it would violate constraint (3). This dictates a counterintuitive situation, namely that in response to slow rotation the superconducting electrons and protons can move only in opposite directions and at different speeds. Their superconducting velocities can be expressed in the following form: $\mathbf{v}_{\alpha}=\gamma_{\alpha} \boldsymbol{\Omega} \times \mathbf{r}$. To find $\gamma_{\alpha}$ we first observe that from the stationarity requirement we can obtain an extra condition by equating the rotation-induced electric current of the normal component (multiplied by -1) to the rotation-induced current response of the superconducting sector subject to constraint (3): $\mathbf{J}_{s}=\left(e_{\mathrm{p}} \gamma_{\mathrm{p}}\left|\Psi_{\mathrm{p}}\right|^{2}+e_{\mathrm{e}} \gamma_{\mathrm{e}}\left|\Psi_{\mathrm{e}}\right|^{2}\right) \boldsymbol{\Omega} \times \mathbf{r}$. From the overall electrical neutrality of the system it follows that the rotation-induced normal current is $\mathbf{J}_{n}=-\left(e_{\mathrm{p}}\left|\Psi_{\mathrm{p}}\right|^{2}+e_{\mathrm{e}}\left|\Psi_{\mathrm{e}}\right|^{2}\right) \boldsymbol{\Omega} \times \mathbf{r}$. Hence we find

$$
\mathbf{v}_{\mathrm{p}}=\frac{\left|\Psi_{\mathrm{p}}\right|^{2}-\left|\Psi_{\mathrm{e}}\right|^{2}}{\left|\Psi_{\mathrm{p}}\right|^{2}+\frac{m_{\mathrm{p}}}{m_{\mathrm{e}}}\left|\Psi_{\mathrm{e}}\right|^{2}} \boldsymbol{\Omega} \times \mathbf{r} ; \quad \mathbf{v}_{\mathrm{e}}=\frac{\left|\Psi_{\mathrm{e}}\right|^{2}-\left|\Psi_{\mathrm{p}}\right|^{2}}{\left|\Psi_{\mathrm{e}}\right|^{2}+\frac{m_{\mathrm{e}}}{m_{\mathrm{p}}}\left|\Psi_{\mathrm{p}}\right|^{2}} \boldsymbol{\Omega} \times \mathbf{r} .
$$

To sustain these counter-currents a rotating two-gap superconductor should generate in its bulk a vector potential and hence rotation induces a magnetic field:

$$
\mathbf{B}_{\mathrm{rot}}=\frac{2}{e} \frac{\left|\Psi_{\mathrm{p}}\right|^{2}-\left|\Psi_{\mathrm{e}}\right|^{2}}{\frac{\left|\Psi_{\mathrm{p}}\right|^{2}}{m_{\mathrm{p}}}+\frac{\left|\Psi_{\mathrm{e}}\right|^{2}}{m_{\mathrm{e}}}} \boldsymbol{\Omega}
$$

Whereas in the bulk the superfluid electrons and protons have the velocities $\mathbf{v}_{e, p}$, the field $\mathbf{B}_{\text {rot }}$ is generated by velocity variations in the layer near the system's edge with thickness of the penetration length $\lambda=\left(e^{2}\left[\left|\Psi_{\mathrm{p}}\right|^{2} / m_{\mathrm{p}}+\left|\Psi_{\mathrm{e}}\right|^{2} / m_{\mathrm{e}}\right]\right)^{-1 / 2}$. This follows from the equation for magnetic field variation, namely $-\lambda^{2} \nabla^{2} \mathbf{B}(\mathbf{r})+\mathbf{B}(\mathbf{r})=\mathbf{B}_{\text {rot }}$. Equation (4) demonstrates a remarkable circumstance: the London law in the two-component superconductor is actually violated. The rotation-induced field is not a universal function of the fundamental constants irrespective of microscopic details. Indeed, it acquires a dependence on densities. At temperatures just below the superconducting transition for protons a rotating sample of radius $R$ generates a magnetic flux of the order of $R^{2} \Omega$ flux quanta ( $R$ given in $\mathrm{cm}$ and $\Omega$ in $\mathrm{s}^{-1}$ ), which could be detectable with modern superconducting quantum interference devices even for samples as small as $10 \mu \mathrm{m}$ rotating at $1 \mathrm{~Hz}$ (we note that it is easier to achieve high pressures in small samples, which makes it a very convenient experimental probe). Going to a larger sample or higher rotation frequency would even allow measurement of the ratio of the condensate densities and their temperature dependences, as follows from (4). Moreover, of course, its absence would even rule out protonic superconductivity or deuteronic condensation. It follows that a direct experimental route exists for the verification of this possible new class of dissipationless states.

Though this has been cast in terms of a possible failure of London's law (otherwise rigorously applicable up to relativistic corrections in electronic superconductors), the major issue discussed here might well be viewed as a possible extension of the classifications of the rotational responses of quantum fluids. Rotational response is a quintessentially state-defining property of quantum fluids, and the one we find in $\mathrm{LMH}$ (as summarized in Fig. 2) is seen to be quite complex; it involves both co- and counter-directed electrical currents, and in particular a current in the direction opposite to rotation. This suggests a classification of the projected liquid state of metallic hydrogen as a new quantum fluid, and one that may present considerable opportunity for new and emerging physics.

Received 15 January 2007; accepted 24 May 2007; published 17 June 2007.

References

1. London, F. Superfluids (Wiley, New York, 1950).

2. Onsager, L. Statistical hydrodynamics. Nuovo Cimento, Suppl. 6, 279-287 (1949).

3. Feynman, R. P. Progress in Low Temperature Physics (North-Holland, Amsterdam, 1955).

4. Andreev, A. F. \& Lifshitz, I. M. Quantum theory of defects in crystals. Sov. Phys. JETP 29, $1107-1113$ (1969).

5. Volovik, G. The Universe in a Helium Droplet (Oxford Univ. Press, USA, 2003).

6. Andreev, A. F. \& Bashkin, E. P. Three-velocity hydrodynamics of superfluid solutions. Sov. Phys. JETP 42, 164-171 (1975)

7. Alpar, M. A., Langer, S. A. \& Sauls, J. A. Rapid postglitch spin-up of the superfluid core in pulsars. Astr. J. 282, 533-541 (1984).

8. Verheijen, A. A., van Ruitenbeek, J. M., de Bruyn Ouboter, A. \& de Jongh, L. J. Measurement of the London moment in two high-temperature superconductors. Nature 345, 418-419 (1990).

9. Ashcroft, N. W. Hydrogen at high density. J. Phys. A: Math. Gen. 36, 6137-6147 (2003).

10. Ashcroft, N. W. Hydrogen dominant metallic alloys: High temperature superconductors? Phys. Rev. Lett. 92, 187002 (2004).

11. Jones, P. B. Type I and two-gap superconductivity in neutron star magnetism. Mon. Not. Roy. Astron. Soc. 371, 1327-1333 (2006).

12. Babaev, E. Vortices with fractional flux in two-gap superconductors and in extended Faddeev model. Phys. Rev. Lett. 89, 067001 (2002).

13. Babaev, E. Phase diagram of planar $\mathrm{U}(1) \mathrm{xU}(1)$ superconductors: Condensation of vortices with fractional flux and a superfluid state. Nucl. Phys. B 686, 397-412 (2004).

14. Babaev, E., Sudbø, A. \& Ashcroft, N. W. A superconductor to superfluid phase transition in liquid metallic hydrogen. Nature 431, 666-669 (2004).

15. Smørgrav, E., Babaev, E., Smiseth, J. \& Sudbø, A. Observation of a metallic superfluid in a numerical experiment. Phys. Rev. Lett. 95, 135301 (2005).

16. Wessel, S. \& Troyer, M. Supersolid hard-core bosons on the triangular lattice. Phys. Rev. Lett. 95, 127205 (2005)

Acknowledgements

This work was supported by the National Science Foundation under grants DMR-0601461 and DMR-0302347.

Correspondence and requests for materials should be addressed to E.B.

Competing financial interests

The authors declare no competing financial interests.

Reprints and permission information is available online at http://npg.nature.com/reprintsandpermissions/ 